\title{
On the Structure of Lie Pseudo-Groups
}

\author{
Peter J. OLVER ${ }^{\dagger}$, Juha POHJANPELTO ${ }^{\ddagger}$ and Francis VALIQUETTE ${ }^{\dagger}$ \\ $\dagger$ School of Mathematics, University of Minnesota, Minneapolis, MN 55455, USA \\ E-mail: olver@math.umn.edu,valiq001@math.umn.edu \\ URL: http://www.math.umn.edu/ olver/, http://www.math.umn.edu/ valiq001/ \\ $\ddagger$ Department of Mathematics, Oregon State University, Corvallis, OR 97331, USA \\ E-mail: juha@math.oregonstate.edu \\ URL: http://oregonstate.edu/ pohjanpp/
}

Received March 31, 2009, in final form July 08, 2009; Published online July 23, 2009

doi:10.3842/SIGMA.2009.077

\begin{abstract}
We compare and contrast two approaches to the structure theory for Lie pseudogroups, the first due to Cartan, and the second due to the first two authors. We argue that the latter approach offers certain advantages from both a theoretical and practical standpoint.
\end{abstract}

Key words: Lie pseudo-group; infinitesimal generator; jet; contact form; Maurer-Cartan form; structure equations; essential invariant

2000 Mathematics Subject Classification: 58A15; 58H05

\section{Introduction}

The aim of this paper is to compare the structure theory for Lie pseudo-groups developed by the first two authors in [16] with the classical Cartan theory, [3, 4, 5]. The former relies on the contact structure of the infinite diffeomorphism jet bundle, whereas Cartan's is based on the prolongation of exterior differential systems. We show that the two theories are isomorphic in the case of transitive Lie pseudo-groups, but lead to different structure equations when dealing with intransitive pseudo-group actions. We then argue that the former theory offers some distinct advantages over the Cartan structure theory in this situation.

Our reference point is the well-established structure theory for finite-dimensional Lie groups. Let $G$ be a Lie group of dimension $r=\operatorname{dim} G$. The commutators

$$
\left[\mathbf{v}_{j}, \mathbf{v}_{k}\right]=\sum_{i=1}^{r} C_{j k}^{i} \mathbf{v}_{i}
$$

between the infinitesimal generators - that is, a basis $\mathbf{v}_{1}, \ldots, \mathbf{v}_{r}$ for its Lie algebra $\mathfrak{g}$ - prescribe the structure constants $C_{j k}^{i}=-C_{k j}^{i}$, which serve to uniquely characterize a connected Lie group $G$ up to a discrete subgroup. Equivalently, the structure of $G$ can be based on the Maurer-Cartan structure equations

$$
d \mu^{i}=-\sum_{j<k} C_{j k}^{i} \mu^{j} \wedge \mu^{k}
$$

satisfied by the Maurer-Cartan one-forms $\mu^{1}, \ldots, \mu^{r}$, which form the dual basis of the dual space $\mathfrak{g}^{*}$. It is noteworthy that the same structure constants appear in both the Maurer-Cartan structure equations and the commutator relations.

\footnotetext{
*This paper is a contribution to the Special Issue "Élie Cartan and Differential Geometry". The full collection is available at http://www.emis.de/journals/SIGMA/Cartan.html
} 
The key obstruction hindering an immediate generalization of the finite-dimensional structure theory to infinite-dimensional Lie pseudo-groups is the lack of an appropriate abstract object to represent the pseudo-group itself. Thus, at least in our current state of knowledge, Lie pseudo-groups are inextricably bound to the manifold on which they act. The appropriate Maurer-Cartan forms thus must be suitably invariant differential forms living on the manifold or, better, on some bundle associated with the action. The approach developed in [16] is based on the bundle of infinite order jets of pseudo-group transformations and the invariant contact forms thereon.

More specifically, the bundle of infinite jets of local diffeomorphisms belonging to the pseudogroup forms a subbundle - indeed a subgroupoid - of the infinite diffeomorphism jet bundle. The Maurer-Cartan forms will be identified as the right-invariant ${ }^{1}$ contact forms on the latter jet bundle, and their structure equations are readily found. Restricting the diffeomorphisminvariant contact forms to the pseudo-group jet subbundle results in a system of MaurerCartan forms for the pseudo-group, whose structure equations are obtained by restriction of the diffeomorphism structure equations. Remarkably, the restricted invariant contact forms, which are no longer linearly independent, satisfy a collection of linear algebraic constraints that can be immediately obtained by lifting the infinitesimal determining equations for the pseudogroup. This allows us to immediately establish a complete system of structure equations for any Lie pseudo-group directly from its infinitesimal determining equations, thereby avoiding the more cumbersome and unintuitive prolongation construction advocated by Cartan. We emphasize that the method does not rely on the explicit formulas for the Maurer-Cartan forms, and only needs elementary linear algebra to extract the complete structure equations. Moreover, the construction can be readily implemented in any coordinate system on the underlying manifold, and avoids having to identify the invariants and work in the specially adapted coordinates as required by Cartan's method. As a result, the theory can be immediately applied in a broad range of examples, and the necessary algorithms are straightforwardly implemented using standard symbolic software packages such as MATHEMATICA or MAPLE.

Another advantage of the contact form approach is that it applies equally well to both transitive and intransitive pseudo-groups, and naturally includes finite-dimensional Lie transformation groups as a particular case. In the transitive case, we show that the Cartan structure equations are isomorphic to those satisfied by the invariant contact forms. However, a direct isomorphism is no longer valid in the more challenging case of intransitive pseudo-group actions. Furthermore, the Maurer-Cartan structure equations established here are directly dual to the commutator equations for the infinitesimal generators of the pseudo-group, and, moreover, coincide with the structure equations (1.2) when the pseudo-group is of finite type. This is in contrast to Cartan's version, which, in particular, produces nonzero structure constants/functions for intransitive actions of abelian pseudo-group and Lie group actions, [23], thus making the connections between the structure equations and the Lie algebra of infinitesimal generators somewhat obscure.

One of the main results of Cartan is that any Lie pseudo-group, after a finite number of prolongations, is characterized by leaving a coframe and a certain number of functions invariant. By virtue of the Cartan-Kähler theorem, [2, 15], Cartan's structure equations serve as integrability conditions on the invariant coframe. The invariant coframe constructed by Cartan depends on the realization of the pseudo-group action, and two pseudo-groups that are isomorphic in the sense of Cartan, [4, 5, 22], can have non-isomorphic Cartan structure equations. On the other hand, our Maurer-Cartan structure equations are always isomorphic under Cartan's notion of isomorphism of pseudo-groups, [23, 24].

Pertinent references on the general theory of Lie pseudo-groups include the classical works of Lie, [11, 12], Cartan, [3, 4, 5], and Vessiot, [25], along with a variety of contemporary treatments,

\footnotetext{
${ }^{1}$ Alternatively, one can use the left-invariant forms. As in the references, for specificity, we focus on the right-invariant constructions here.
} 
$[6,7,8,9,10,13,16,19,21,22]$. The basics of jet bundles, contact forms, and the variational bicomplex can be found, for instance, in [1, 15]. Applications of these results in the method of moving frames for pseudo-groups can be found in [17, 18].

\section{The diffeomorphism pseudo-group}

We begin by describing the structure of the most basic pseudo-group. Let $M$ be a smooth $m$ dimensional manifold and write $\mathcal{D}=\mathcal{D}(M)$ for the pseudo-group of all local ${ }^{2}$ diffeomorphisms $\varphi: M \rightarrow M$. For each $0 \leq n \leq \infty$, let $\mathcal{D}^{(n)} \subset J^{n}(M, M)$ denote the bundle of their $n$-th order jets. We remark that $\mathcal{D}^{(n)}$ carries the structure of a groupoid, [14], whose multiplication is provided by algebraic composition of Taylor series (when defined). There are natural right and left actions of $\mathcal{D}$ on the jet bundles $\mathcal{D}^{(n)}$, denoted by $R_{\varphi}$ and $L_{\varphi}$, respectively.

Local coordinates $\left(z, Z^{(n)}\right)$ on $\mathcal{D}^{(n)}$ are provided by a system of source coordinates $z=$ $\left(z^{1}, \ldots, z^{m}\right)$ on $M$, target coordinates $Z=\left(Z^{1}, \ldots, Z^{m}\right)$ also on $M$, and jet coordinates $Z_{A}^{b}$ representing the partial derivatives $\partial^{k} \varphi^{b}(z) / \partial z^{a_{1}} \cdots \partial z^{a_{k}}$ of the local diffeomorphism $Z=\varphi(z)$. Here $A=\left(a_{1}, \ldots, a_{k}\right)$, with $1 \leq a_{\nu} \leq m$, indicates a multi-index of order $k=\# A \leq n$. In what follows, we will consistently use lower case letters, $z, x, u, \ldots$ for the source coordinates and the corresponding upper case letters $Z, X, U, \ldots$ for the target coordinates.

At infinite order, the cotangent bundle $T^{\star} \mathcal{D}^{(\infty)} \subset T^{\star} J^{\infty}(M, M)$ naturally splits into horizontal and vertical (contact) components, spanned respectively by the coordinate differentials $d z^{1}, \ldots, d z^{m}$, and the basic contact forms

$$
\Upsilon_{A}^{b}=d Z_{A}^{b}-\sum_{a=1}^{m} Z_{A, a}^{b} d z^{a}, \quad b=1, \ldots, m, \quad \# A \geq 0 .
$$

The decomposition of $T^{\star} \mathcal{D}^{(\infty)}$ accordingly splits the differential $d=d_{M}+d_{G}$, where the subscript on the vertical differential $d_{G}$ refers to the groupoid structure of $\mathcal{D}^{(\infty)}$. In particular, if $F\left(z, Z^{(n)}\right)$ is any differential function, then

$$
d_{M} F=\sum_{a=1}^{m}\left(\mathbb{D}_{z^{a}} F\right) d z^{a}, \quad d_{G} F=\sum_{b=1}^{m} \sum_{\# A \geq 0} \frac{\partial F}{\partial Z_{A}^{b}} \Upsilon_{A}^{b},
$$

where

$$
\mathbb{D}_{z^{a}}=\frac{\partial}{\partial z^{a}}+\sum_{b=1}^{m} \sum_{\# A \geq 0} Z_{A, a}^{b} \frac{\partial}{\partial Z_{A}^{b}}, \quad a=1, \ldots, m,
$$

denotes the coordinate total derivative operators.

Since the target coordinate functions $Z^{a}: \mathcal{D}^{(\infty)} \rightarrow \mathbb{R}$ are clearly invariant under the right action of $\mathcal{D}$, so are their differentials $d Z^{a}$. The splitting of the differential into horizontal and contact components is also right-invariant. This implies that the one-forms

$$
\sigma^{a}=d_{M} Z^{a}=\sum_{b=1}^{m} Z_{b}^{a} d z^{b}, \quad a=1, \ldots, m,
$$

form an invariant horizontal coframe, while

$$
\mu^{a}=d_{G} Z^{a}=\Upsilon^{a}=d Z^{a}-\sum_{b=1}^{m} Z_{b}^{a} d z^{b}, \quad a=1, \ldots, m,
$$

\footnotetext{
${ }^{2}$ Our notational conventions allow the domain of definition of a map $\varphi: M \rightarrow M$ to be a proper open subset: $\operatorname{dom} \varphi \subset M$. Also, when we write $Z=\varphi(z)$ we implicitly assume $z \in \operatorname{dom} \varphi$.
} 
are the zero-th order invariant contact forms. Writing the horizontal component of the differential of a differential function $F: \mathcal{D}^{(\infty)} \rightarrow \mathbb{R}$ in terms of the invariant horizontal coframe (2.3),

$$
d_{M} F=\sum_{a=1}^{m}\left(\mathbb{D}_{Z^{a}} F\right) \sigma^{a},
$$

serves to define the dual invariant total differential operators

$$
\mathbb{D}_{Z^{a}}=\sum_{b=1}^{m} w_{a}^{b} \mathbb{D}_{z^{b}}, \quad a=1, \ldots, m,
$$

where

$$
\left(w_{a}^{b}\left(z, Z^{(1)}\right)\right)=\left(\frac{\partial Z^{b}}{\partial z^{a}}\right)^{-1}
$$

denotes the inverse Jacobian matrix. Thus, higher-order right-invariant contact forms are obtained by successively applying the invariant differential operators (2.5) to the zero-th order invariant contact forms (2.4):

$$
\mu_{A}^{a}=\mathbb{D}_{Z}^{A} \mu^{a}=\mathbb{D}_{Z^{a_{1}}} \cdots \mathbb{D}_{Z^{a_{k}}} \mu^{a}, \quad a=1, \ldots, m, \quad \# A \geq 0 .
$$

The differential operators $\mathbb{D}_{Z^{1}}, \ldots, \mathbb{D}_{Z^{m}}$ mutually commute, so the order of differentiation in (2.6) is immaterial. As in [16], we interpret the right-invariant contact forms $\mu^{(\infty)}=$ $\left(\ldots \mu_{A}^{a} \ldots\right)$ as the Maurer-Cartan forms for the diffeomorphism pseudo-group $\mathcal{D}$, and they, together with the horizontal forms (2.3) provide a right-invariant coframe on $\mathcal{D}^{(\infty)}$.

The diffeomorphism structure equations satisfied by the Maurer-Cartan forms are easily established, [16]. They can be concisely expressed by introducing the vector-valued MaurerCartan formal power series $\mu \llbracket H \rrbracket=\left(\mu^{1} \llbracket H \rrbracket, \ldots, \mu^{m} \llbracket H \rrbracket\right)^{T}$, with components

$$
\mu^{a} \llbracket H \rrbracket=\sum_{\# A \geq 0} \frac{1}{A !} \mu_{A}^{a} H^{A}, \quad a=1, \ldots, m .
$$

Here $H=\left(H^{1}, \ldots, H^{m}\right)$ are formal power series parameters, while $A !=i_{1} ! i_{2} ! \cdots i_{m}$ !, where $i_{l}$ stands for the number of occurrences of the integer $l$ in $A$. The structure equations for the right-invariant forms $\mu_{A}^{a}$ are obtained by comparing the coefficients of the various powers of $H$ in the power series identity

$$
d \mu \llbracket H \rrbracket=\nabla \mu \llbracket H \rrbracket \wedge(\mu \llbracket H \rrbracket-d Z),
$$

where $d Z=\left(d Z^{1}, \ldots, d Z^{m}\right)^{T}$, and where $\nabla \mu \llbracket H \rrbracket=\left(\partial \mu^{a} \llbracket H \rrbracket / \partial H^{b}\right)$ denotes the $m \times m$ Jacobian matrix obtained by formal differentiation of the power series (2.7) with respect to the parameters. The complete structure equations for the diffeomorphism pseudo-group are then furnished by equations (2.8) together with the equations

$$
d \sigma=\nabla \mu \llbracket 0 \rrbracket \wedge \sigma
$$

for the invariant horizontal forms $\sigma=\left(\sigma^{1}, \ldots, \sigma^{m}\right)^{T}$. We restrict the structure equations (2.8) to a target fiber $\left(\boldsymbol{\tau}^{(\infty)}\right)^{-1}(Z) \subset \mathcal{D}^{(\infty)}$ to obtain the Maurer-Cartan structure equations for the diffeomorphism pseudo-group. This amounts to setting

$$
0=d Z=\sigma+\mu \llbracket 0 \rrbracket, \quad \text { so that } \quad \sigma=-\mu \llbracket 0 \rrbracket \text {. }
$$

Consequently, the structure equations (2.9) for the horizontal forms $\sigma$ become identical with the structure equations for the zero-th order Maurer-Cartan forms $\mu^{a}=\mu^{a} \llbracket 0 \rrbracket$. 
Theorem 2.1. The Maurer-Cartan structure equations for the diffeomorphism pseudo-group $\mathcal{D}$ are

$$
d \mu \llbracket H \rrbracket=\nabla \mu \llbracket H \rrbracket \wedge \mu \llbracket H \rrbracket .
$$

Example 2.2. For the pseudo-group $\mathcal{D}(\mathbb{R})$ of local diffeomorphisms of $M=\mathbb{R}$, the MaurerCartan power series is

$$
\mu \llbracket H \rrbracket=\sum_{n=0}^{\infty} \mu_{n} \frac{H^{n}}{n !},
$$

where $\mu_{n}=\mathbb{D}_{X}^{n} \mu_{0}, n=0,1,2 \ldots$, are the right-invariant contact forms on $\mathcal{D}^{(\infty)}$. The individual components of (2.11) yield the expressions

$$
d \mu_{n}=\sum_{i=0}^{n}\left(\begin{array}{c}
n \\
i
\end{array}\right) \mu_{i+1} \wedge \mu_{n-i}=-\sum_{i=0}^{[(n+1) / 2]} \frac{n-2 i+1}{n+1}\left(\begin{array}{c}
n+1 \\
i
\end{array}\right) \mu_{i} \wedge \mu_{n+1-i}, \quad n \geq 0,
$$

which reproduce the structure equations found by Cartan, [4, equation (48)].

Expanding the power series (2.7), we find that the Maurer-Cartan structure equations (2.11) have the individual components

$$
d \mu_{C}^{a}=\sum_{C=(A, B)} \sum_{b=1}^{m}\left(\begin{array}{l}
C \\
A
\end{array}\right) \mu_{A, b}^{a} \wedge \mu_{B}^{b},
$$

involving the multinomial coefficients

$$
\left(\begin{array}{l}
C \\
A
\end{array}\right)=\frac{C !}{A ! B !} \quad \text { when } \quad C=(A, B)=\left(a_{1}, \ldots, a_{k}, b_{1}, \ldots, b_{l}\right)
$$

is the union of two multi-indices $A=\left(a_{1}, \ldots, a_{k}\right)$ and $B=\left(b_{1}, \ldots, b_{l}\right)$, either of which can be empty.

Remark 2.3. Since the higher order Maurer-Cartan forms $\mu_{C}^{a}$ are defined by (2.6), their structure equations (2.12) can also be derived by Lie differentiating the structure equations for the zero-th order invariant contact forms $\mu^{a}$. By direct computation

$$
d \mu^{a}=\sum_{b=1}^{m} \mu_{b}^{a} \wedge\left(\mu^{b}-d Z^{b}\right)
$$

and, from the Leibniz rule, we obtain

$$
\begin{aligned}
d \mu_{C}^{a}=d\left(\mathbb{D}_{Z}^{C} \mu^{a}\right)=\mathbb{D}_{Z}^{C}\left(d \mu^{a}\right) & =\mathbb{D}_{Z}^{C}\left(\sum_{b=1}^{m} \mu_{b}^{a} \wedge\left(\mu^{b}-d Z^{b}\right)\right) \\
& =\sum_{C=(A, B)} \sum_{b=1}^{m}\left(\begin{array}{l}
C \\
A
\end{array}\right) \mu_{A, b}^{a} \wedge\left(\mu_{B}^{b}-d\left(\mathbb{D}_{Z}^{B} Z^{b}\right)\right) .
\end{aligned}
$$

The last term, $d\left(\mathbb{D}_{Z}^{B} Z^{b}\right)$, is non-trivial only when $\# B=0$. Restricting the last equation to a target fiber $\left(\boldsymbol{\tau}^{(\infty)}\right)^{-1}(Z)$ we recover the Maurer-Cartan structure equations (2.12). 


\section{$3 \quad$ Lie pseudo-groups}

Let $\mathcal{G} \subset \mathcal{D}$ be a sub-pseudo-group acting on $M$ and let $\mathcal{G}^{(n)} \subset \mathcal{D}^{(n)}, 0 \leq n \leq \infty$, denote the corresponding subgroupoid of $n$-th order jets of its local diffeomorphisms. Roughly speaking, $\mathcal{G}$ is called a Lie pseudo-group provided that it can be identified as the solution space to a system of partial differential equations. There are several variants of the precise technical requirements to be found in the literature; ours are the following.

Definition 3.1. A sub-pseudo-group $\mathcal{G} \subset \mathcal{D}$ is called a Lie pseudo-group if there exists $n^{\star} \geq 1$ such that, for all finite $n \geq n^{\star}$ :

1. $\mathcal{G}^{(n)} \subset \mathcal{D}^{(n)}$ forms a smooth, embedded subbundle;

2. $\pi_{n}^{n+1}: \mathcal{G}^{(n+1)} \rightarrow \mathcal{G}^{(n)}$ is a fibration;

3. if $j_{n} \varphi \subset \mathcal{G}^{(n)}$, then $\varphi \in \mathcal{G}$;

4. $\mathcal{G}^{(n)}=\operatorname{pr}^{\left(n-n^{\star}\right)} \mathcal{G}^{\left(n^{\star}\right)}$ is obtained by prolongation.

The minimal value of $n^{\star}$ is called the order of the Lie pseudo-group.

Thus, by condition 1 , the pseudo-group jet subbundle $\mathcal{G}^{(n)} \subset \mathcal{D}^{(n)}$ is prescribed in local coordinates by a system of $n$-th order (typically nonlinear) partial differential equations

$$
F^{(n)}\left(z, Z^{(n)}\right)=0
$$

known as the $n$-th order determining equations for the Lie pseudo-group $\mathcal{G}$. By construction, for any $n \geq n^{\star}$, the system (3.1) is locally solvable, and its local solutions $Z=\varphi(z)$, by condition 3 , are precisely the pseudo-group transformations. Moreover, by condition 4 , the determining equations in order $n>n^{\star}$ can be obtained by repeatedly applying the total derivative operators (2.2) to those of order $n^{\star}$.

Let $\mathfrak{g}$ denote the local Lie algebra ${ }^{3}$ of infinitesimal generators of our pseudo-group $\mathcal{G}$, i.e., the set of locally defined vector fields whose flow maps belong to the pseudo-group. Let $J^{n} \mathfrak{g} \subset$ $J^{n} T M, 0 \leq n \leq \infty$, denote their $n$-jets. Fiber coordinates on the vector field jet bundle $J^{n} T M$ are given by $\zeta_{A}^{b}$, for $1 \leq b \leq m, 0 \leq \# A \leq n$, representing the partial derivatives $\partial^{k} \zeta^{b}(z) / \partial z^{a_{1}} \cdots \partial z^{a_{k}}$ of the components of a vector field

$$
\mathbf{v}=\sum_{a=1}^{m} \zeta^{a}(z) \frac{\partial}{\partial z^{a}}
$$

written in local coordinate $z=\left(z^{1}, \ldots, z^{m}\right)$ on $M$. By linearizing the $n$-th order pseudo-group determining equations (3.1) at the $n$-jet of the identity transformation, we see that the subbundle $J^{n} \mathfrak{g} \subset J^{n} T M$ can locally be viewed as a system of linear partial differential equations

$$
L^{(n)}\left(z, \zeta^{(n)}\right)=0,
$$

for the coefficients $\zeta(z)=\left(\zeta^{1}(z), \ldots, \zeta^{m}(z)\right)$ of the infinitesimal generators, called the infinitesimal determining equations of the pseudo-group. In particular, if $\mathcal{G}$ arises as the symmetry pseudo-group of a system of partial differential equations, then (3.3) is the involutive completion of the usual system of determining equations resulting from applying Lie's infinitesimal symmetry algorithm, [15].

The Maurer-Cartan forms associated with the Lie pseudo-group $\mathcal{G}$ are obtained by pulling back the diffeomorphism Maurer-Cartan forms (2.6) to the pseudo-group jet bundle $\mathcal{G}^{(\infty)} \subset \mathcal{D}^{(\infty)}$.

\footnotetext{
${ }^{3}$ By "local Lie algebra", we mean that the vector fields $\mathbf{v} \in \mathfrak{g}$ may only be locally defined, and that, for $\mathbf{v}, \mathbf{w} \in \mathfrak{g}$, the Lie bracket $[\mathbf{v}, \mathbf{w}] \in \mathfrak{g}$ is only defined on their common domain of definition.
} 
The restricted Maurer-Cartan forms are, of course, no longer linearly independent. However, the induced dependencies can, perhaps surprisingly, be explicitly prescribed with the aid of the infinitesimal determining equations, [16].

Theorem 3.2. The complete set of linear dependencies among the right-invariant MaurerCartan forms $\mu^{(\infty)}$ is provided by the linear system

$$
L^{(\infty)}\left(Z, \mu^{(\infty)}\right)=0
$$

obtained from the linear determining equations (3.3) by replacing the source variables $z^{a}$ by the corresponding target variables $Z^{a}$, and the infinitesimal generator jet coordinates $\zeta_{A}^{b}$ by the corresponding Maurer-Cartan forms $\mu_{A}^{b}$.

The equations (3.4) are called the lifted infinitesimal determining equations for the Lie pseudogroup $\mathcal{G}$. (See [16] for additional details on the lifting process.) Thus, the structure equations for our pseudo-group can simply be obtained by restricting the diffeomorphism structure equations (2.11) to the solution space to the lifted infinitesimal determining equations (3.4).

Theorem 3.3. The Maurer-Cartan structure equations of a Lie pseudo-group $\mathcal{G}$ are obtained by imposing the linear relations prescribed by the lifted infinitesimal determining equations (3.4) on the diffeomorphism Maurer-Cartan equations (2.11):

$$
\left.(d \mu \llbracket H \rrbracket=\nabla \mu \llbracket H \rrbracket \wedge \mu \llbracket H \rrbracket)\right|_{L^{(\infty)}\left(Z, \mu^{(\infty)}\right)=0} .
$$

Remark 3.4. The motivation behind the need to restrict the Maurer-Cartan forms to a target fiber can be readily understood in the context of finite-dimensional Lie group actions. In this situation, $\boldsymbol{\tau}^{(\infty)}: \mathcal{G}^{(\infty)} \rightarrow M$ will typically be a principal $G$ bundle, and, consequently, the independent Maurer-Cartan forms on $\mathcal{G}^{(\infty)}$ and their structure equations, when restricted to a target fiber $\left(\boldsymbol{\tau}^{(\infty)}\right)^{-1}(Z) \simeq G$ coincide with the usual Maurer-Cartan forms and their structure equations (1.2).

However, it is worth pointing out that, due to the appearance of the coordinates $z$ in the infinitesimal determining equations (3.3), the basis of $\mathfrak{g}^{\star}$ prescribed by the independent restricted invariant contact forms $\mu_{A}^{b}$ may vary from fiber to fiber as the target point $Z$ ranges over $M$. Consequently, the structure coefficients in the pseudo-group structure equations (3.5) may very well be $Z$-dependent. It is noteworthy that, when $\mathcal{G}$ is of finite type and so represents the action of a finite-dimensional Lie group $G$ on $M$, the resulting variable structure coefficients $C_{j k}^{i}(Z)$ represent the same Lie algebra $\mathfrak{g}$ and so are all similar, modulo a $Z$-dependent change of basis, to the usual constant structure coefficients associated with a fixed basis of $\mathfrak{g}^{\star}$. In contrast, this is not necessarily the case for infinite-dimensional intransitive pseudo-group actions. The non-constant invariants (under change of Maurer-Cartan basis) of the structure coefficients are essential invariants, first exposed by Cartan, [3, 22]; see [23, 24] for further details, comparing Cartan's approach with ours. The existence of essential invariants is one of the key obstacles preventing the construction of a suitable abstract object representing the pseudo-group.

\section{Cartan structure equations}

In this section we provide a brief overview of Cartan's method for constructing the structure equations for a Lie pseudo-group. For more detailed accounts, we refer the reader to Cartan's original works $[4,5]$, and to the expository texts [8, 22].

Given a Lie pseudo-group $\mathcal{G}$ acting on $M$, we choose local coordinates

$$
z=(x, y)=\left(x^{1}, \ldots, x^{s}, y^{1}, \ldots, y^{t}\right)=\left(z^{1}, \ldots, z^{m}\right), \quad s+t=m=\operatorname{dim} M,
$$


so that the pseudo-group action takes the form

$$
X^{i}=x^{i}, \quad Y^{\alpha}=f^{\alpha}(x, y), \quad i=1, \ldots, s, \quad \alpha=1, \ldots, t,
$$

with $\operatorname{det}\left(\partial f^{\alpha} / \partial y^{\beta}\right) \neq 0$. Thus, the $x^{i}$ are invariants of the action, whose common level sets prescribe the $t$-dimensional pseudo-group orbits in $M$. Let

$$
X=x, \quad F^{\left(n_{\star}\right)}\left(x, y, Y^{\left(n_{\star}\right)}\right)=0,
$$

be the involutive determining equations. We note that $n_{\star} \geq n^{\star}$, the order of the pseudo-group, as the minimal order determining equations might need to be completed to involution, [20].

For any $1 \leq n \leq \infty$, let $\mathcal{C}^{(n)}$ denote the contact system on $\mathcal{D}^{(n)}$ spanned by the contact forms $\Upsilon_{A}^{b}$ of order $0 \leq \# A<n,(2.1)$. The first step in Cartan's procedure is to restrict the contact system $\mathcal{C}^{\left(n_{\star}\right)}$ to the subbundle $\mathcal{G}^{\left(n_{\star}\right)} \subset \mathcal{D}^{\left(n_{\star}\right)}$. The aim is to recast the determining system (4.2) in terms of the Pfaffian system

$$
\begin{aligned}
& X^{i}-x^{i}=0, \quad i=1, \ldots, s, \quad \alpha=1, \ldots, t, \quad 0 \leq \# A \leq n_{\star}-1, \\
& \left.\Upsilon_{A}^{s+\alpha}\right|_{F^{\left(n_{\star}\right)}\left(x, y, Y^{\left(n_{\star}\right)}\right)=0}=\left.\left(d Y_{A}^{\alpha}-\sum_{b=1}^{m} Y_{A, b}^{\alpha} d z^{b}\right)\right|_{F^{\left(n_{\star}\right)}\left(x, y, Y^{\left(n_{\star}\right)}\right)=0}=0 .
\end{aligned}
$$

For $k \geq 1$, let $Y_{[k]}=\left(Y_{[k]}^{1}, \ldots, Y_{[k]}^{t_{k}}\right)$ be local parameterizations for the fibers of the bundles $\mathcal{G}^{(k)} \rightarrow \mathcal{G}^{(k-1)}$, where $t_{k}=\operatorname{dim} \mathcal{G}^{(k)}-\operatorname{dim} \mathcal{G}^{(k-1)}$ is the fiber dimension. The system (4.3) is then equivalent to

$$
\begin{aligned}
& X^{i}-x^{i}=0, \quad i=1, \ldots, s, \\
& d Y^{\alpha}-\sum_{a=1}^{m} L_{a}^{\alpha}\left(z, Y, Y_{[1]}\right) d z^{a}=0, \quad \alpha=1, \ldots, t, \\
& d Y_{[1]}^{j}-\sum_{a=1}^{m} L_{[1], a}^{j}\left(z, Y, Y_{[1]}, Y_{[2]}\right) d z^{a}=0, \quad j=1, \ldots, t_{1}, \\
& \quad \vdots \\
& d Y_{\left[n_{\star}-1\right]}^{k}-\sum_{a=1}^{m} L_{\left[n_{\star}-1\right], a}^{k}\left(z, Y, Y_{[1]}, \ldots, Y_{\left[n_{\star}\right]}\right) d z^{a}=0, \quad k=1, \ldots, t_{n_{\star}-1},
\end{aligned}
$$

where the functions $L_{a}^{\alpha}, \ldots, L_{\left[n_{\star}-1\right], a}^{i}$ are prescribed by the determining system (4.2). With the differential forms (4.4) in hand, Cartan proceeds, in an inductive manner, to derive a system of invariant one-forms that serve to characterize the pseudo-group.

Since the forms $d Y^{1}, \ldots, d Y^{t}$ are right-invariant and the action of $\mathcal{G}$ on $\mathcal{G}^{\left(n_{\star}\right)}$ preserves the contact system $\mathcal{C}^{\left(n_{\star}\right)}$, the forms

$$
\omega_{[0]}^{s+\alpha}=\sum_{a=1}^{m} L_{a}^{\alpha}\left(z, Y, Y_{[1]}\right) d z^{a}, \quad \alpha=1, \ldots, t,
$$

must likewise be right-invariant. These together with the invariant forms

$$
\omega_{[0]}^{i}=d x^{i}, \quad i=1, \ldots, s,
$$

constitute a basis of horizontal forms, and hence $d z^{1}, \ldots, d z^{m}$ can be expressed as linear combinations of $\omega_{[0]}^{1}, \ldots, \omega_{[0]}^{m}$. Their exterior derivatives have the form

$$
d \omega_{[0]}^{b}=\sum_{a=1}^{m} d L_{a}^{b}\left(z, Y, Y_{[1]}\right) \wedge d z^{a}=\sum_{a=1}^{m} \omega_{[0]}^{a} \wedge \pi_{a}^{b}, \quad b=1, \ldots, m,
$$


where the one-forms $\pi_{a}^{b}$ are certain linear combinations of $\omega_{[0]}^{1}, \ldots, \omega_{[0]}^{m}, d Y^{1}, \ldots, d Y^{t}$, and $d Y_{[1]}^{1}, \ldots, d Y_{[1]}^{t_{1}}$. The invariance of $\omega_{[0]}^{1}, \ldots, \omega_{[0]}^{m}$ implies that

$$
\sum_{a=1}^{m} \omega_{[0]}^{a} \wedge\left(R_{\psi}^{\star}\left(\pi_{a}^{b}\right)-\pi_{a}^{b}\right)=0, \quad b=1, \ldots, m
$$

for all $\psi \in \mathcal{G}$ such that the pull-back $R_{\psi}^{\star}\left(\pi_{a}^{b}\right)$ is defined. This means that

$$
R_{\psi}^{\star}\left(\pi_{a}^{b}\right) \equiv \pi_{a}^{b} \quad \bmod \omega_{[0]}^{1}, \ldots, \omega_{[0]}^{m} .
$$

By the assumptions, $t_{1}=\operatorname{dim} \mathcal{G}^{(1)}-\operatorname{dim} \mathcal{G}^{(0)}$ of the $\pi_{a}^{b}$ are linearly independent modulo $\omega_{[0]}^{1}, \ldots$, $\omega_{[0]}^{m}, d Y^{1}, \ldots, d Y^{t}$. Those $t_{1}$ differential forms can be written as

$$
\pi^{i} \equiv \sum_{j=1}^{t_{1}} c_{j}^{i} d Y_{[1]}^{j}+\sum_{\alpha=1}^{q} e_{\alpha}^{i} d Y^{\alpha} \quad \bmod \omega_{[0]}^{1}, \ldots, \omega_{[0]}^{m}, \quad i=1, \ldots, t_{1},
$$

with $\operatorname{det}\left(c_{j}^{i}\right) \neq 0$. The coefficients $c_{j}^{i}$ and $e_{\alpha}^{i}$ may depend on the variables $z, Y$, and $Y_{[1]}$. By adding suitable multiples of the $\omega_{[0]}^{a}$ we can write

$$
\pi^{i} \equiv \omega_{[1]}^{i} \quad \bmod \omega_{[0]}^{1}, \ldots, \omega_{[0]}^{m}, \quad i=1, \ldots, t_{1},
$$

where

$$
\omega_{[1]}^{i}:=\sum_{j=1}^{t_{1}} c_{j}^{i}\left(d Y_{[1]}^{j}-\sum_{b=1}^{m} L_{[1], b}^{j}\left(z, Y, Y_{[1]}\right) d z^{b}\right)+\sum_{\alpha=1}^{q} e_{\alpha}^{i}\left(d Y^{\alpha}-\omega_{[0]}^{p+\alpha}\right) .
$$

Cartan, [4, pp. 597-600], now concludes that the one-forms $\omega_{[1]}^{1}, \ldots \omega_{[1]}^{t_{1}}$, are right-invariant. These first order Cartan forms are equivalent to our first order Maurer-Cartan forms (2.6) in the sense that

$$
\operatorname{span}\left\{\omega_{[1]}^{1}, \ldots, \omega_{[1]}^{t_{1}}\right\}=\operatorname{span}\left\{\left.\mu_{Z^{b}}^{a}\right|_{L^{\left(n_{\star}\right)}\left(Z, \mu^{\left(n_{\star}\right)}\right)=0}\right\} .
$$

Next by computing the exterior derivatives of the first order Cartan forms (4.6) and repeating the above procedure, Cartan derives $t_{2}$ linearly independent invariant second order Cartan forms, and so on, up to order $n_{\star}-1$.

The $r_{n_{\star}-1}=m+t_{1}+t_{2}+\cdots+t_{n_{\star}-1}$ invariant one-forms so constructed are collectively denoted by $\omega^{1}, \omega^{2}, \ldots, \omega^{r_{\star}-1}$ without the subscripts. Their exterior derivatives can be written as

$$
d \omega^{i}=\sum_{1 \leq j<k \leq r_{n_{\star}-1}} C_{j k}^{i} \omega^{j} \wedge \omega^{k}+\sum_{j=1}^{r_{n_{\star}-1}} \sum_{\beta=1}^{t_{n_{\star}}} A_{j \beta}^{i} \omega^{j} \wedge \bar{\pi}^{\beta}, \quad i=1, \ldots, r_{n_{\star}-1},
$$

where

$$
\left(\bar{\pi}^{1}, \ldots, \bar{\pi}^{t_{n_{\star}}}\right) \equiv\left(d Y_{\left[n_{\star}\right]}^{1}, \ldots, d Y_{\left[n_{\star}\right]}^{t_{n_{\star}}}\right) \quad \bmod \omega^{1}, \ldots, \omega^{r_{n^{\star}-1}}
$$

These constitute Cartan's structure equations. If the pseudo-group acts intransitively, the structure coefficients $C_{j k}^{i}, A_{j \beta}^{i}$ may depend on the invariants $x^{1}, \ldots, x^{s}$. 


\section{Examples}

In this section we illustrate the two structure theories with a pair of elementary intransitive Lie pseudo-group actions.

Example 5.1. Let $\mathcal{G}$ be the infinite-dimensional Lie pseudo-group

$$
X=x, \quad Y=y f(x)+\phi(x), \quad Z=z(f(x))^{x}+\psi(x),
$$

where $f, \phi, \psi \in C^{\infty}(\mathbb{R})$ and $f(x)>0$. This Lie pseudo-group was introduced by Cartan, [3], as an example with an essential invariant.

We first construct the structure equations using Cartan's structure theory. The involutive determining system is

$$
\begin{aligned}
& X=x, \quad Y_{z}=0, \quad Z_{y}=0, \quad Z_{z}=\left(Y_{y}\right)^{x}, \quad Y_{y y}=0, \\
& Z_{z z}=0, \quad Z_{z x}=\left(Y_{y}\right)^{x}\left(\log Y_{y}+\frac{x Y_{x y}}{Y_{y}}\right) .
\end{aligned}
$$

Thus, the fibers of the bundle $\pi_{0}^{2}: \mathcal{G}^{(2)} \rightarrow \mathcal{G}^{(0)}$ are parameterized by

$$
\left(Y_{x}, Y_{y}, Z_{x}, Y_{x x}, Y_{x y}, Z_{x x}\right)
$$

and the determining system (5.1) is equivalent to the Pfaffian system

$$
\begin{array}{ll}
X-x=0, & \\
\left.\Upsilon^{y}\right|_{\mathcal{G}^{(2)}}=d Y-Y_{x} d x-Y_{y} d y=0, & \left.\Upsilon^{z}\right|_{\mathcal{G}^{(2)}}=d Z-Z_{x} d x-\left(Y_{y}\right)^{x} d z=0, \\
\left.\Upsilon_{x}^{y}\right|_{\mathcal{G}^{(2)}}=d Y_{x}-Y_{x x} d x-Y_{x y} d y=0, & \left.\Upsilon_{y}^{y}\right|_{\mathcal{G}^{(2)}}=d Y_{y}-Y_{y x} d x=0, \\
\left.\Upsilon_{x}^{z}\right|_{\mathcal{G}^{(2)}}=d Z_{x}-Z_{x x} d x-\left(Y_{y}\right)^{x}\left(\log Y_{y}+\frac{x Y_{x y}}{Y_{y}}\right) d z=0 .
\end{array}
$$

Cartan's algorithm yields the six invariant one-forms

$$
\begin{aligned}
\omega^{1} & =d x, \quad \omega^{2}=Y_{x} d x+Y_{y} d y, \quad \omega^{3}=Z_{x} d x+\left(Y_{y}\right)^{x} d z, \\
\omega^{4} & =\left.\mu_{X}^{y}\right|_{\mathcal{G}^{(2)}}=\frac{1}{Y_{y}} \Upsilon_{y}^{y}, \quad \omega^{5}=\left.\mu_{Y}^{y}\right|_{\mathcal{G}^{(2)}}=\Upsilon_{x}^{y}-\frac{Y_{x}}{Y_{y}} \Upsilon_{y}^{y}, \\
\omega^{6} & =\left.\mu_{X}^{z}\right|_{\mathcal{G}^{(2)}}=\Upsilon_{x}^{z}-\frac{x Z_{x}}{Y_{y}} \Upsilon_{y}^{y} .
\end{aligned}
$$

By computing their exterior derivatives we obtain Cartan's structure equations

$$
\begin{aligned}
& d \omega^{1}=0, \quad d \omega^{2}=\omega^{4} \wedge \omega^{1}+\omega^{5} \wedge \omega^{2}, \quad d \omega^{3}=\omega^{6} \wedge \omega^{1}+x \omega^{5} \wedge \omega^{3}, \\
& d \omega^{4}=\omega^{1} \wedge \bar{\pi}^{1}+\omega^{2} \wedge \bar{\pi}^{2}+\omega^{5} \wedge \omega^{4}, \quad d \omega^{5}=\omega^{1} \wedge \bar{\pi}^{2}, \\
& d \omega^{6}=\omega^{1} \wedge \bar{\pi}^{3}+\omega^{3} \wedge\left(\omega^{5}+x \bar{\pi}^{2}\right)+x \omega^{5} \wedge \omega^{6},
\end{aligned}
$$

where

$$
\bar{\pi}^{1}=\left.\mu_{X X}^{y}\right|_{\mathcal{G}^{(2)}}, \quad \bar{\pi}^{2}=\left.\mu_{X Y}^{y}\right|_{\mathcal{G}^{(2)}}, \quad \bar{\pi}^{3}=\left.\mu_{X X}^{z}\right|_{\mathcal{G}^{(2)}} .
$$

On the other hand, the computation of the Maurer-Cartan structure equations by the algorithm presented in Section 3 proceeds as follows. The infinitesimal generators

$$
\mathbf{v}=\xi(x, y, z) \partial_{x}+\eta(x, y, z) \partial_{y}+\zeta(x, y, z) \partial_{z}=[\alpha(x) y+\beta(x)] \partial_{y}+[\alpha(x) x z+\gamma(x)] \partial_{z}
$$


of the pseudo-group action (5.1) are the solutions of the infinitesimal determining system

$$
\xi=0, \quad \eta_{z}=0, \quad \zeta_{y}=0, \quad \zeta_{z}=x \eta_{y},
$$

which can be obtained by linearizing (5.1) at the identity. As in (3.4), the lift of (5.3) produces the linear relations

$$
\mu^{x}=0, \quad \mu_{Z}^{y}=0, \quad \mu_{Y}^{z}=0, \quad \mu_{Z}^{z}=X \mu_{Y}^{y},
$$

among the first order Maurer-Cartan forms. On account of (5.4) and its first prolongation, the structure equations for the zero-th and first order Maurer-Cartan forms are

$$
\begin{aligned}
& d \mu^{y}=\mu_{X}^{y} \wedge \mu^{x}+\mu_{Y}^{y} \wedge \mu^{y}+\mu_{Z}^{y} \wedge \mu^{z}=\mu_{Y}^{y} \wedge \mu^{y}, \\
& d \mu^{z}=\mu_{X}^{z} \wedge \mu^{x}+\mu_{Y}^{z} \wedge \mu^{y}+\mu_{Z}^{z} \wedge \mu^{z}=X \mu_{Y}^{y} \wedge \mu^{z}, \\
& d \mu_{X}^{y}=\mu_{Y}^{y} \wedge \mu_{X}^{y}+\mu_{X Y}^{y} \wedge \mu^{y}, \\
& d \mu_{Y}^{y}=0 \\
& d \mu_{X}^{z}=X \mu_{Y}^{y} \wedge \mu_{X}^{z}+\left(\mu_{Y}^{y}+X \mu_{X Y}^{y}\right) \wedge \mu^{z} .
\end{aligned}
$$

The two sets of structure equations (5.2) and (5.5) are isomorphic provided we set $x=X$ and $\omega^{1}=0$ in Cartan's structure equations (5.2).

Example 5.2. As the second example we consider the action of a one-dimensional Lie group on $\mathbb{R}^{2}$ by translations

$$
X=x \neq 0, \quad Y=y+a x, \quad a \in \mathbb{R} .
$$

Cartan computes the structure equations for this group, [5, p. 1345], and finds

$$
d \omega^{1}=0, \quad d \omega^{2}=\frac{1}{x} \omega^{1} \wedge \omega^{2}
$$

where

$$
\omega^{1}=d x, \quad \omega^{2}=d y-\frac{y}{x} d x .
$$

Equations (5.7) involve two independent invariant one-forms and a non-vanishing, variable structure coefficient. They obviously do not conform with the structure equations for a one-dimensional abelian Lie group.

On the other hand, the Maurer-Cartan structure equations (3.5) for the pseudo-group (5.6) have the desired form. First, the minimal order involutive determining system for the group action (5.6) is

$$
X=x, \quad Y-y=x Y_{x}, \quad Y_{y}=1 .
$$

Linearization of (5.8) yields the infinitesimal determining equations

$$
\xi=0, \quad \eta=x \eta_{x}, \quad \eta_{y}=0,
$$

for the infinitesimal generators $\mathbf{v}=\xi(x, y) \partial_{x}+\eta(x, y) \partial_{y}$. The lift of (5.9) produces the linear relations

$$
\mu^{x}=0, \quad \mu^{y}=X \mu_{X}^{y}, \quad \mu_{Y}^{y}=0,
$$

among the first order Maurer-Cartan forms. It follows from (5.10) that $\mu^{y}$ forms a basis for the Maurer-Cartan forms. Its exterior derivative is given by

$$
d \mu^{y}=\mu_{Y}^{y} \wedge \mu^{y}=0,
$$

which agrees with the structure equation for a one-dimensional abelian Lie group. As in Example 5.1, Cartan's structure equations (5.7) become equivalent with (5.11) once we set $\omega^{1}=0$. 
Since there is no abstract object to represent a pseudo-group, saying when two pseudo-group actions come from the "same pseudo-group" is more tricky than in the finite-dimensional case of Lie group actions. The following definition encapsulates Cartan and Vessiot's notion of isomorphism, [4, 25].

Definition 5.3. Two pseudo-group actions $\mathcal{G}_{1}, \mathcal{G}_{2}$ on manifolds $M_{1}, M_{2}$ are isomorphic, written $\mathcal{G}_{1} \sim \mathcal{G}_{2}$, if they have a common isomorphic prolongation, meaning a pseudo-group $\mathcal{G}$ acting on a manifold $M$, and surjective submersions $\pi_{i}: M \rightarrow M_{i}, i=1,2$, such that, for each $i=1,2$, there is a one-to-one correspondence between elements $\varphi \in \mathcal{G}$ and $\varphi_{i} \in \mathcal{G}_{i}$ satisfying $\pi_{i} \circ \varphi=$ $\varphi_{i} \circ \pi_{i}$.

For example, two actions of the same finite-dimensional Lie group are isomorphic, as one can take $M=M_{1} \times M_{2}$ with the Cartesian product action. Proof of the transitive property of isomorphisms, i.e. $\mathcal{G}_{1} \sim \mathcal{G}_{2}$ and $\mathcal{G}_{2} \sim \mathcal{G}_{3}$, then $\mathcal{G}_{1} \sim \mathcal{G}_{3}$, can be found in Stormark, [22].

On the other hand, two isomorphic pseudo-groups need not have the same Cartan structure equations. A basic illustration of this fact is provided by Example 5.2. Clearly, the group action (5.6) is isomorphic to the group of translations of $\mathbb{R}$,

$$
Y=y+a, \quad a \in \mathbb{R},
$$

which is characterized by the single invariant one-form $\omega=d y$. The Cartan structure equation of the latter action is, of course, $d \omega=0$, which obviously is not isomorphic to the structure equations (5.7). On the other hand, the Maurer-Cartan structure equation of the group action (5.12) is again given by (5.11). In fact, it can be proved, [23, 24], that isomorphic pseudo-group actions always possess isomorphic Maurer-Cartan equations.

The two examples above show that, when dealing with intransitive Lie pseudo-group actions, the Maurer-Cartan structure equations (3.5) and Cartan's structure equations (4.7) do not agree. We refer the reader to [23] for more examples. The discrepancy between the two sets

of structure equations is due to the inclusion of the horizontal forms $\omega_{[0]}^{1}, \ldots, \omega_{[0]}^{s}$, cf. (4.5), in Cartan's version. They do not appear in the Maurer-Cartan structure equations (3.5) since, for a Lie pseudo-group action of the form (4.1), the first $s$ zero-th order Maurer-Cartan forms vanish:

$$
\mu^{i}=0, \quad i=1, \ldots, s .
$$

Restricting to a target fiber yields

$$
\omega_{[0]}^{i}=\sigma^{i}=-\mu^{i}=0, \quad i=1, \ldots, s .
$$

On the other hand, for transitive Lie pseudo-group actions, the two sets of structure equations are equivalent since the relations (2.10) provide a one-to-one correspondence between the zero-th order Maurer-Cartan forms $\mu^{1}, \ldots, \mu^{m}$ and the invariant horizontal forms $\sigma^{1}, \ldots, \sigma^{m}$.

\section{Duality}

In this final section, we investigate the relationship between pseudo-group structure equations and the commutator relations among their infinitesimal generators. As we will see, the MaurerCartan structure equations of Theorem 2.1 are naturally dual to the commutator relations among the infinitesimal generators, in the same sense as the finite-dimensional version in (1.1), (1.2).

Under the identification of infinite jets of local vector fields (3.2) with their Taylor expansions

$$
\left.j_{\infty} \mathbf{v}\right|_{z_{0}} \simeq \sum_{a=1}^{m} \sum_{\# A \geq 0} \zeta_{A}^{a}\left(z_{0}\right) \frac{\left(z-z_{0}\right)^{A}}{A !} \frac{\partial}{\partial z^{a}}
$$


the fiber $\left.J^{\infty} T M\right|_{z_{0}}$ inherits a Lie algebra structure. The monomial vector fields

$$
\mathbf{v}_{a}^{A}=\frac{\left(z-z_{0}\right)^{A}}{A !} \frac{\partial}{\partial z^{a}}, \quad \# A \geq 0, \quad a=1, \ldots, m
$$

provide a basis for the vector space $\left.J^{\infty} T M\right|_{z_{0}}$ and satisfy the Lie bracket relations

$$
\left[\mathbf{v}_{a}^{A}, \mathbf{v}_{b}^{B}\right]=\left(\begin{array}{c}
A, B \backslash a \\
A
\end{array}\right) \mathbf{v}_{b}^{A, B \backslash a}-\left(\begin{array}{c}
B, A \backslash b \\
B
\end{array}\right) \mathbf{v}_{a}^{B, A \backslash b} .
$$

In the above equation

$$
\left(\begin{array}{cc}
A, B \backslash a \\
A
\end{array}\right)= \begin{cases}\frac{(A, B \backslash a) !}{A !(B \backslash a) !}, & a \in B, \\
0, & a \notin B,\end{cases}
$$

where $B \backslash a$ denotes the multi-index obtained by deleting one occurrence of $a$ from $B$. By direct inspection, we conclude that, as in the finite-dimensional theory, the commutation relations (6.1) are directly dual to the Maurer-Cartan structure equations (2.12).

The duality between the Maurer-Cartan structure equations and the Lie brackets of jets of infinitesimal diffeomorphism generators extends straightforwardly to general Lie pseudo-group actions.

Theorem 6.1. The Maurer-Cartan structure equations (3.5) of a Lie pseudo-group $\mathcal{G}$ at a target fiber $\left(\boldsymbol{\tau}^{(\infty)}\right)^{-1}(Z)$ are dual to the Lie algebra structure equations for the fiber $J^{\infty} \mathfrak{g}_{\mid Z}$ of the jet bundle of its infinitesimal generators.

The proof relies on the observation that the Lie algebra structure equations for $J^{\infty} \mathfrak{g}$ are obtained by imposing the constraints prescribed by the infinitesimal determining equations (3.3) on equations (6.1), while the Maurer-Cartan structure equations of a Lie pseudo-group $\mathcal{G} \subset \mathcal{D}$ are, in turn, obtained by imposing the constraints dictated by the lifted version (3.4) of the infinitesimal determining equations on (2.12). The details can be found in [24].

Finally, we note that the horizontal forms $\omega_{[0]}^{i}=d x^{i}, i=1, \ldots, s$, in (4.5) are, naturally, invariant under the group of translations $X^{i}=x^{i}+a^{i}$. Thus Cartan's equations (4.7) more appropriately reflect the infinitesimal structure of the extended set of transformations

$$
X^{i}=x^{i}+b^{i}, \quad Y^{\alpha}=f^{\alpha}(x, y), \quad i=1, \ldots, s, \quad \alpha=1, \ldots, t .
$$

acting transitively on $M$. However, there is no guarantee that the transformations (6.2) represent a Lie pseudo-group. Indeed, for the Lie group action (5.6) of Example 5.2, the extension (6.2) has the form

$$
X=x+b \neq 0, \quad Y=y+a x, \quad a, b \in \mathbb{R},
$$

which does not define a transformation group.

\section{Acknowledgments}

We would like to thank Olle Stormark and anonymous referees for helpful remarks and references that served to improve the paper. The first author was supported in part by NSF Grant DMS 08-07317; the second author by NSF Grant OCE 06-21134; the third author by NSF Grant DMS 05-05293 and a University of Minnesota Graduate School Doctoral Dissertation Fellowship. 


\section{References}

[1] Anderson I.M., The variational bicomplex, Utah State Technical Report, 1989, available at http://www . math. usu .edu/ fg_mp/.

[2] Bryant R.L., Chern S.S., Gardner R.B., Goldschmidt H.L., Griffiths P.A., Exterior differential systems, Mathematical Sciences Research Institute Publications, Vol. 18, Springer-Verlag, New York, 1991.

[3] Cartan É., Sur la structure des groupes infinis, in Oeuvres Complètes, Part II, Vol. 2, Gauthier-Villars, Paris, 1953, 567-569.

[4] Cartan É., Sur la structure des groupes infinis de transformations, in Oeuvres Complètes, Part II, Vol. 2, Gauthier-Villars, Paris, 1953, 571-714.

[5] Cartan É., La structure des groupes infinis, in Oeuvres Complètes, Part II, Vol. 2, Gauthier-Villars, Paris, 1953, 1335-1384.

[6] Chrastina J., The formal theory of differential equations, Masaryk University, Brno, 1998.

[7] Ehresmann C., Introduction à la théorie des structures infinitésimales et des pseudo-groupes de Lie, in Géometrie Différentielle, Colloques Internationaux du Centre National de la Recherche Scientifique, Strasbourg, 1953, 97-110.

[8] Kamran N., Contributions to the study of the equivalence problem of Élie Cartan and its applications to partial and ordinary differential equations, Acad. Roy. Belg. Cl. Sci. Mem. Collect. 8o (2) 45 (1989), no. 7, 122 pages.

[9] Kuranishi M., On the local theory of continuous infinite pseudo-groups. I, Nagoya Math. J. 15 (1959), $225-260$.

[10] Kuranishi M., On the local theory of continuous infinite pseudo-groups. II, Nagoya Math. J. 19 (1961), $55-91$.

[11] Lie S., Über unendlichen kontinuierliche Gruppen, Christ. Forh. Aar. 8 (1883), 1-47 (see also Gesammelte Abhandlungen, Vol. 5, B.G. Teubner, Leipzig, 1924, 314-360).

[12] Lie S., Die Grundlagen für die Theorie der unendlichen kontinuierlichen Transformationsgruppen, Leipzig. Ber. 43 (1891), 316-393 (see also Gesammelte Abhandlungen, Vol. 6, B.G. Teubner, Leipzig, 1927, 300-364).

[13] Lisle I.G., Reid G.J., Cartan structure of infinite Lie pseudogroups, in Geometric Approaches to Differential Equations (Canberra, 1995), Editors P.J. Vassiliou and I.G. Lisle, Austral. Math. Soc. Lect. Ser., Vol. 15, Cambridge University Press, Cambridge, 2000, 116-145.

[14] Mackenzie K., Lie groupoids and Lie algebroids in differential geometry, London Mathematical Society Lecture Note Series, Vol. 124, Cambridge University Press, Cambridge, 1987.

[15] Olver P.J., Equivalence, invariants, and symmetry, Cambridge University Press, Cambridge, 1995.

[16] Olver P.J., Pohjanpelto J., Maurer-Cartan equations and structure of Lie pseudo-groups, Selecta Math. (N.S.) 11 (2005), 99-126.

[17] Olver P.J., Pohjanpelto J., Moving frames for Lie pseudo-groups, Canad. J. Math. 60 (2008), $1336-1386$.

[18] Olver P.J., Pohjanpelto J., Differential invariant algebras of Lie pseudo-groups, Adv. Math., to appear.

[19] Pommaret J.-F., Systems of partial differential equations and Lie pseudogroups, Mathematics and Its Applications, Vol. 14, Gordon \& Breach Science Publishers, New York, 1978.

[20] Seiler W.M., Involution - the formal theory of differential equations and its applications in computer algebra and numerical analysis, Habilitation Thesis, Dept. of Mathematics, Universität Mannheim, 2002.

[21] Singer I., Sternberg S., The infinite groups of Lie and Cartan. I. The transitive groups, J. Analyse Math. 15 (1965), 1-114.

[22] Stormark O., Lie's structural approach to PDE systems, Encyclopedia of Mathematics and Its Applications, Vol. 80, Cambridge University Press, Cambridge, 2000.

[23] Valiquette F., Structure equations of Lie pseudo-groups, J. Lie Theory 18 (2008), 869-895.

[24] Valiquette F., Applications of moving frames to Lie pseudo-groups, Ph.D. Thesis, University of Minnesota, 2009.

[25] Vessiot E., Sur la théorie des groupes continues, Ann. École Norm. Sup. 20 (1903), 411-451. 